\title{
Necessary Condition for an Euler-Lagrange Equation on Time Scales
}

\author{
Monika Dryl and Delfim F. M. Torres \\ Center for Research and Development in Mathematics and Applications (CIDMA), Department of Mathematics, \\ University of Aveiro, 3810-193 Aveiro, Portugal
}

Correspondence should be addressed to Delfim F. M. Torres; delfim@ua.pt

Received 28 January 2014; Accepted 13 March 2014; Published 7 April 2014

Academic Editor: Ryan Loxton

Copyright (C) 2014 M. Dryl and D. F. M. Torres. This is an open access article distributed under the Creative Commons Attribution License, which permits unrestricted use, distribution, and reproduction in any medium, provided the original work is properly cited.

We prove a necessary condition for a dynamic integrodifferential equation to be an Euler-Lagrange equation. New and interesting results for the discrete and quantum calculus are obtained as particular cases. An example of a second order dynamic equation, which is not an Euler-Lagrange equation on an arbitrary time scale, is given.

\section{Introduction}

The time-scale calculus is a unification of the theories of difference and differential equations, unifying integral and differential calculus with the calculus of finite differences and offering a formalism for studying hybrid discrete-continuous dynamical systems $[1,2]$. It has applications in any field that requires simultaneous modeling of discrete and continuous data [3-5].

The study of optimal control problems on arbitrary time scales is a subject under strong current research $[6,7]$. This is particularly true for the particular, but rich, case of the calculus of variations on time scales [8-10]. Compared with the direct problem, that establishes dynamic equations of EulerLagrange type to the time-scale variational problems, the inverse problem has not yet been studied in the framework of time scales. It turns out that there is a simple explanation for the absence of such an inverse general theory for the time-scale variational calculus: the classical approach relies on the use of the chain rule, which is not valid in the general context of time scales [2]. To address the problem, a different approach to the subject is needed.

In this paper we introduce a completely different approach to the inverse problem of the calculus of variations, using an integral perspective instead of the classical differential point of view [11, 12]. The differential form of equations is often related to dynamics via the time derivative. The integral form has proved to be successful for proving the existence and uniqueness of solutions, to study analytical properties of solutions, and to prove coherence of variational embeddings [13]. Here we show its usefulness with respect to the inverse problem of the calculus of variations. We prove a necessary condition for an integrodifferential equation on an arbitrary time scale $\mathbb{T}$ to be an Euler-Lagrange equation, related to a property of self-adjointness (Definition 12) of the equation of variation (Definition 13) of the given dynamic integrodifferential equation.

The text is organized as follows. Section 2 provides all the necessary definitions and results of the delta calculus on time scales, which will be used throughout the text. The main results are proved in Section 3. We present a suffi- cient condition of self-adjointness for an integrodifferential equation (Lemma 15). Using this property, we prove a necessary condition for a general (nonclassical) inverse problem of the calculus of variations on an arbitrary time scale (Theorem 16). As a result, we obtain a useful tool to identify integrodifferential equations which are not Euler-Lagrange equations (Remark 17). To illustrate the method, we give a second order dynamic equation on time scales which is not an EulerLagrange equation (Example 19). Next we apply Theorem 16 to the particular cases of time scales $\mathbb{\mathbb { T }} \in\left\{\mathbb{R}, h \mathbb{Z}, \overline{q^{\mathbb{Z}}}\right\}, h>0$, 
$q>1$ (Corollaries 20, 21, and 22). In Section 4 some final remarks are presented. We begin by proving the equivalence between an integrodifferential equation and a second order dynamic equation (Proposition 23). Then we show that, due to lack of a chain rule on an arbitrary time scale, it is impossible to obtain an equivalence between equations of variation in integral and differential forms. This is in contrast with the classical case $\mathbb{T}=\mathbb{R}$, where such equivalence holds (Proposition 24).

\section{Preliminaries}

In this section we introduce basic definitions and theorems that will be useful in the sequel. For more results concerning the theory of time scales we refer the reader to the books [2, $4]$.

Definition 1 (e.g., Section 2.1 of [14]). A time scale $\mathbb{T}$ is an arbitrary nonempty closed subset of $\mathbb{R}$. Given a time scale $\mathbb{T}$, the forward jump operator $\sigma: \mathbb{T} \rightarrow \mathbb{T}$ is defined by $\sigma(t):=\inf \{s \in \mathbb{T}: s>t\}$ for $t \neq \sup \mathbb{T}$ and $\sigma(\sup \mathbb{\mathbb { V }}):=$ sup $\mathbb{T}$ if sup $\mathbb{Z}<+\infty$. Similarly, the backward jump operator $\rho: \mathbb{T} \rightarrow \mathbb{T}$ is defined by $\rho(t):=\sup \{s \in \mathbb{T}: s<t\}$ for $t \neq \inf \mathbb{T}$ and $\rho(\inf \mathbb{T})=\inf \mathbb{T}$ if inf $\mathbb{T}>-\infty$.

A point $t \in \mathbb{T}$ is called right-dense, right-scattered, leftdense, or left-scattered if $\sigma(t)=t, \sigma(t)>t, \rho(t)=t$, and $\rho(t)<t$, respectively. The forward graininess function $\mu$ : $\mathbb{T} \rightarrow[0, \infty)$ is defined by $\mu(t):=\sigma(t)-t$. To simplify the notation, one usually uses $f^{\sigma}(t):=f(\sigma(t))$.

The delta derivative is defined for points from the set

$$
\mathbb{T}^{\mathcal{K}}:= \begin{cases}\mathbb{T} \backslash\{\sup \mathbb{\mathbb { }}\} & \text { if } \rho(\sup \mathbb{T})<\sup \mathbb{T}<\infty, \\ \mathbb{T} & \text { otherwise. }\end{cases}
$$

Definition 2 (Section 1.1 of [2]). Let $f: \mathbb{T} \rightarrow \mathbb{R}$ and $t \in \mathbb{T}^{\kappa}$. One defines $f^{\Delta}(t)$ to be the number (provided it exists) with the property that, given any $\varepsilon>0$, there is a neighborhood $U$ of $t$ such that

$$
\left|f^{\sigma}(t)-f(s)-f^{\Delta}(t)(\sigma(t)-s)\right| \leq \varepsilon|\sigma(t)-s| \quad \forall s \in U .
$$

We call $f^{\Delta}(t)$ the delta derivative of $f$ at $t$. Function $f$ is delta differentiable on $\mathbb{T}^{\kappa}$ provided $f^{\Delta}(t)$ exists for all $t \in \mathbb{T}^{\kappa}$. Then, $f^{\Delta}: \mathbb{T}^{\kappa} \rightarrow \mathbb{R}$ is called the delta derivative of $f$ on $\mathbb{T}^{\kappa}$.

Theorem 3 (Theorem 1.16 of [2]). Let $f: \mathbb{T} \rightarrow \mathbb{R}$ and $t \in \mathbb{T}^{\mathcal{K}}$. If $f$ is continuous at $t$ and $t$ is right-scattered, then $f$ is delta differentiable at $t$ with

$$
f^{\Delta}(t)=\frac{f^{\sigma}(t)-f(t)}{\mu(t)} .
$$

Theorem 4 (Theorem 1.20 of [2]). Let $f, g: \mathbb{T} \rightarrow \mathbb{R}$ be delta differentiable at $t \in \mathbb{T}^{\mathcal{k}}$. Then,

(1) the sum $f+g: \mathbb{T} \rightarrow \mathbb{R}$ is delta differentiable at $t$ with

$$
(f+g)^{\Delta}(t)=f^{\Delta}(t)+g^{\Delta}(t) ;
$$

(2) for any real constant $\alpha, \alpha f: \mathbb{T} \rightarrow \mathbb{R}$ is delta differentiable at $t$ with

$$
(\alpha f)^{\Delta}(t)=\alpha f^{\Delta}(t)
$$

(3) the product $f g: \mathbb{T} \rightarrow \mathbb{R}$ is delta differentiable at $t$ with

$$
\begin{aligned}
(f g)^{\Delta}(t) & =f^{\Delta}(t) g(t)+f^{\sigma}(t) g^{\Delta}(t) \\
& =f(t) g^{\Delta}(t)+f^{\Delta}(t) g^{\sigma}(t) .
\end{aligned}
$$

Theorem 5 (Theorem 1.16 of [2]). If $f: \mathbb{T} \rightarrow \mathbb{R}$ is a delta differentiable function at $t, t \in \mathbb{T}^{\kappa}$, then

$$
f^{\sigma}(t)=f(t)+\mu(t) f^{\Delta}(t) .
$$

Definition 6 (Definition 1.58 of [2]). A function $f: \mathbb{T} \rightarrow \mathbb{R}$ is called rd-continuous provided it is continuous at right-dense points in $\mathbb{T}$ and its left-sided limits exist (finite) at all leftdense points in $\mathbb{T}$.

The set of all rd-continuous functions $f: \mathbb{T} \rightarrow \mathbb{R}$ is denoted by $C_{\mathrm{rd}}=C_{\mathrm{rd}}(\mathbb{T})=C_{\mathrm{rd}}(\mathbb{T}, \mathbb{R})$. The set of functions $f: \mathbb{T} \rightarrow \mathbb{R}$ that are delta differentiable and whose derivative is rd-continuous is denoted by $C_{\mathrm{rd}}^{1}=C_{\mathrm{rd}}^{1}(\mathbb{T})=C_{\mathrm{rd}}^{1}(\mathbb{T}, \mathbb{R})$.

Definition 7 (Definition 1.71 of [2]). A function $F: \mathbb{T} \rightarrow \mathbb{R}$ is called an antiderivative of $f: \mathbb{T} \rightarrow \mathbb{R}$ provided $F^{\Delta}(t)=f(t)$ for all $t \in \mathbb{T}^{\kappa}$.

Definition 8. Let $\mathbb{T}$ be a time scale and $a, b \in \mathbb{T}$. If $f$ : $\mathbb{T}^{\kappa} \rightarrow \mathbb{R}$ is a rd-continuous function and $F: \mathbb{T} \rightarrow \mathbb{R}$ is an antiderivative of $f$, then the delta integral is defined by

$$
\int_{a}^{b} f(t) \Delta t:=F(b)-F(a) .
$$

Theorem 9 (Theorem 1.74 of [2]). Every rd-continuous function $f$ has an antiderivative $F$. In particular, if $t_{0} \in \mathbb{T}$, then $F$ defined by

$$
F(t):=\int_{t_{0}}^{t} f(\tau) \Delta \tau, \quad t \in \mathbb{T},
$$

is an antiderivative of $f$.

Example 10. Let $a, b \in \mathbb{T}$ and $f: \mathbb{T} \rightarrow \mathbb{R}$ be rd-continuous. If $\mathbb{T}=\mathbb{R}$, then

$$
\int_{a}^{b} f(t) \Delta t=\int_{a}^{b} f(t) d t
$$

where the integral on the right side is the usual Riemann integral. If $\mathbb{T}=h \mathbb{Z}, h>0$, then

$$
\int_{a}^{b} f(t) \Delta t= \begin{cases}\sum_{k=a / h}^{(b / h)-1} f(k h) h, & \text { if } a<b, \\ 0, & \text { if } a=b, \\ -\sum_{k=(b / h)}^{(a / h)-1} f(k h) h, & \text { if } a>b .\end{cases}
$$


If $\mathbb{T}=\overline{q^{\mathbb{Z}}}, q>1$, and $a<b$, then

$$
\int_{a}^{b} f(t) \Delta t=(q-1) \sum_{t \in[a, b) \cap \mathbb{T}} t f(t)
$$

Theorem 11 (Theorem 1.77 of [2]). If $a, b, c \in \mathbb{T}, \alpha \in \mathbb{R}$, and $f, g \in C_{r d}(\mathbb{T})$, then

(1) $\int_{a}^{b}[f(t)+g(t)] \Delta t=\int_{a}^{b} f(t) \Delta t+\int_{a}^{b} g(t) \Delta t$;

(2) $\int_{a}^{b}(\alpha f)(t) \Delta t=\alpha \int_{a}^{b} f(t) \Delta t$;

(3) $\int_{a}^{b} f(t) g^{\Delta}(t) \Delta t=(f g)(b)-(f g)(a)-\int_{a}^{b} f^{\Delta}(t) g^{\sigma}(t) \Delta t$;

(4) $\int_{a}^{b} f^{\sigma}(t) g^{\Delta}(t) \Delta t=(f g)(b)-(f g)(a)-\int_{a}^{b} f^{\Delta}(t) g(t) \Delta t$.

For more properties of the delta derivative and delta integral we refer the reader to $[2,4]$.

\section{Main Results}

Our main result (Theorem 16) provides a necessary condition for an integrodifferential equation on an arbitrary time scale to be an Euler-Lagrange equation. For that the notions of self-adjointness (Definition 12) and equation of variation (Definition 13) are essential. These definitions, in integrodifferential form, are new (cf. the notion of self-adjointness for a dynamic time-scale equation of second order in [2, Section 4.1] and the notion of equation of variation for a second order differential equation in [12]).

Definition 12 (first order self-adjoint integrodifferential equation). A first order integrodifferential dynamic equation is said to be self-adjoint if it has the form

$$
L u(t)=\text { const, }
$$

where $L u(t)=p(t) u^{\Delta}(t)+\int_{t_{0}}^{t}\left[q(s) u^{\sigma}(s)\right] \Delta s$,

with $p, q \in C_{\mathrm{rd}}, p \neq 0$ for all $t \in \mathbb{T}$, and $t_{0} \in \mathbb{T}$.

Let $\mathbb{D}$ be the set of all functions $y: \mathbb{T} \rightarrow \mathbb{R}$ such that $y^{\Delta}: \mathbb{T}^{\kappa} \rightarrow \mathbb{R}$ is continuous. A function $y \in \mathbb{D}$ is said to be a solution of (13) provided $L y(t)=$ const holds for all $t \in \mathbb{T}^{\kappa}$. Along the text we use the operators $[\cdot]_{\mathbb{T}}$ and $\langle\cdot\rangle_{\mathbb{T}}$ defined by

$$
\begin{gathered}
{[y]_{\mathbb{T}}(t):=\left(t, y^{\sigma}(t), y^{\Delta}(t)\right),} \\
\langle y\rangle_{\mathbb{T}}(t):=\left(t, y^{\sigma}(t), y^{\Delta}(t), y^{\Delta \Delta}(t)\right) .
\end{gathered}
$$

Definition 13 (equation of variation). Let

$$
H[y]_{\mathbb{V}}(t)+\int_{t_{0}}^{t} G[y]_{\mathbb{V}}(s) \Delta s=\mathrm{const}
$$

be an integrodifferential equation on time scales with $\partial_{3} H \neq 0$ and $t \rightarrow \partial_{2} F[y]_{\mathbb{T}}(t), t \rightarrow \partial_{3} F[y]_{\mathbb{T}}(t) \in C_{\text {rd }}$ along every curve $y$, where $F \in\{G, H\}$. The equation of variation associated with (15) is given by

$$
\begin{aligned}
\partial_{2} H[u]_{\mathbb{T}} & (t) u^{\sigma}(t)+\partial_{3} H[u]_{\mathbb{T}}(t) u^{\Delta}(t) \\
& +\int_{t_{0}}^{t} \partial_{2} G[u]_{\mathbb{T}}(s) u^{\sigma}(s)+\partial_{3} G[u]_{\mathbb{T}}(s) u^{\Delta}(s) \Delta s=0 .
\end{aligned}
$$

Remark 14. The equation of variation (16) can be interpreted in the following way. Assuming $y=y(t, b), b \in \mathbb{R}$, is a oneparameter solution of a given integrodifferential equation (15), then

$$
\begin{aligned}
& H\left(t, y^{\sigma}(t, b), y^{\Delta}(t, b)\right) \\
& \quad+\int_{t_{0}}^{t} G\left(s, y^{\sigma}(s, b), y^{\Delta}(s, b)\right) \Delta s=\text { const. }
\end{aligned}
$$

Let $u(t)$ be a particular solution; that is, $u(t)=y(t, \bar{b})$ for a certain $\bar{b}$. Differentiating (17) with respect to the parameter $b$ and then putting $b=\bar{b}$, we obtain (16).

Lemma 15 (sufficient condition of self-adjointness). Let (15) be a given integrodifferential equation. If

$$
\partial_{2} H[y]_{\mathbb{T}}(t)+\partial_{3} G[y]_{\mathbb{T}}(t)=0,
$$

then its equation of variation (16) is self-adjoint.

Proof. Let us consider a given equation of variation (16). Using Theorem 5 and third item of Theorem 11, we expand the two components of the given equation:

$$
\begin{gathered}
\partial_{2} H[u]_{\mathbb{T}}(t) u^{\sigma}(t)=\partial_{2} H[u]_{\mathbb{T}}(t)\left(u(t)+\mu(t) u^{\Delta}(t)\right), \\
\int_{t_{0}}^{t} \partial_{3} G[u]_{\mathbb{T}}(s) u^{\Delta}(s) \Delta s \\
=\partial_{3} G[u]_{\mathbb{T}}(t) u(t)-\partial_{3} G[u]_{\mathbb{T}}\left(t_{0}\right) u\left(t_{0}\right) \\
-\int_{t_{0}}^{t}\left[\partial_{3} G[u]_{\mathbb{T}}(s)\right]^{\Delta} u^{\sigma}(s) \Delta s .
\end{gathered}
$$

Hence, equation of variation (16) can be written in the form

$$
\begin{aligned}
\partial_{3} G[u]_{\mathbb{T}}\left(t_{0}\right) u\left(t_{0}\right) & \\
= & u^{\Delta}(t)\left[\mu(t) \partial_{2} H[u]_{\mathbb{T}}(t)+\partial_{3} H[u]_{\mathbb{T}}(t)\right] \\
& +\int_{t_{0}}^{t} u^{\sigma}(s)\left[\partial_{2} G[u]_{\mathbb{T}}(s)-\left(\partial_{3} G[u]_{\mathbb{T}}(s)\right)^{\Delta}\right] \Delta s \\
& +u(t)\left(\partial_{2} H[u]_{\mathbb{T}}(t)+\partial_{3} G[u]_{\mathbb{T}}(t)\right) .
\end{aligned}
$$

If (18) holds, then (20) is a particular case of (13) with

$$
\begin{gathered}
p(t)=\mu(t) \partial_{2} H[u]_{\mathbb{T}}(t)+\partial_{3} H[u]_{\mathbb{T}}(t), \\
q(s)=\partial_{2} G[u]_{\mathbb{T}}(s)-\left(\partial_{3} G[u]_{\mathbb{T}}(s)\right)^{\Delta}, \\
\partial_{3} G[u]_{\mathbb{T}}\left(t_{0}\right) u\left(t_{0}\right)=\text { const. }
\end{gathered}
$$

This concludes the proof. 
Theorem 16 (necessary condition for an Euler-Lagrange equation in integral form). Let $\mathbb{T}$ be an arbitrary time scale and let

$$
H\left(t, y^{\sigma}(t), y^{\Delta}(t)\right)+\int_{t_{0}}^{t} G\left(s, y^{\sigma}(s), y^{\Delta}(s)\right) \Delta s=\text { const }
$$

be a given integrodifferential equation. If (22) is to be an EulerLagrange equation, then its equation of variation (16) is selfadjoint, in the sense of Definition 12.

Proof. Assume (22) is the Euler-Lagrange equation of the variational functional

$$
\mathscr{I}(y)=\int_{t_{0}}^{t_{1}} L\left(t, y^{\sigma}(t), y^{\Delta}(t)\right) \Delta t
$$

where $L \in C^{2}$. Since the Euler-Lagrange equation in integral form of (23) is given by

$$
\partial_{3} L[y]_{\mathbb{T}}(t)+\int_{t_{0}}^{t}-\partial_{2} L[y]_{\mathbb{T}}(s) \Delta s=\mathrm{const}
$$

(cf. $[13,15,16])$, we conclude that $H[y]_{\mathbb{T}}(t)=\partial_{3} L[y]_{\mathbb{T}}(t)$ and $G[y]_{\mathbb{T}}(s)=-\partial_{2} L[y]_{\mathbb{T}}(s)$. Having in mind that

(i) $\partial_{2} H=\partial_{2}\left(\partial_{3} L\right), \partial_{3} H=\partial_{3}\left(\partial_{3} L\right)=\partial_{3}^{2} L$,

(ii) $\partial_{2} G=\partial_{2}\left(-\partial_{2} L\right)=-\partial_{2}^{2} L, \partial_{3} G=\partial_{3}\left(-\partial_{2} L\right)=-\partial_{3} \partial_{2} L$,

it follows from Schwarz's theorem, $\partial_{2} \partial_{3} L=\partial_{3} \partial_{2} L$, that

$$
\partial_{2} H[y]_{\mathbb{T}}(t)+\partial_{3} G[y]_{\mathbb{T}}(t)=0
$$

We conclude from Lemma 15 that the equation of variation (22) is self-adjoint.

Remark 17. In practical terms, Theorem 16 is useful to identify equations which are not Euler-Lagrange equations: if the equation of variation (16) of a given dynamic equation (15) is not self-adjoint, then we conclude that (15) is not an EulerLagrange equation.

Remark 18 (self-adjointness for a second order differential equation). Let $p$ be delta differentiable in Definition 12 and $u \in C_{\text {rd }}^{2}$. Then, by differentiating (13), one obtains a second order self-adjoint dynamic equation

$$
p^{\sigma}(t) u^{\Delta \Delta}(t)+p^{\Delta}(t) u^{\Delta}(t)+q(t) u^{\sigma}(t)=0
$$

or

$$
p(t) u^{\Delta \Delta}(t)+p^{\Delta}(t) u^{\Delta \sigma}(t)+q(t) u^{\sigma}(t)=0
$$

with $q \in C_{\mathrm{rd}}$ and $p \in C_{\mathrm{rd}}^{1}$ and $p \neq 0$ for all $t \in \mathbb{T}$.

Now we present an example of a second order differential equation on time scales which is not an Euler-Lagrange equation.
Example 19. Let us consider the following second order dynamic equation on an arbitrary time scale $\mathbb{T}$ :

$$
y^{\Delta \Delta}(t)+y^{\Delta}(t)-t=0 .
$$

We may write (28) in integrodifferential form (15):

$$
y^{\Delta}(t)+\int_{t_{0}}^{t}\left(y^{\Delta}(s)-s\right) \Delta s=\text { const }
$$

where $H[y]_{\mathbb{T}}(t)=y^{\Delta}(t)$ and $G[y]_{\mathbb{T}}(t)=y^{\Delta}(t)-t$. Because

$$
\begin{aligned}
& \partial_{2} H[y]_{\mathbb{T}}(t)=\partial_{2} G[y]_{\mathbb{T}}(t)=0, \\
& \partial_{3} H[y]_{\mathbb{T}}(t)=\partial_{3} G[y]_{\mathbb{T}}(t)=1,
\end{aligned}
$$

the equation of variation associated with (29) is given by

$$
u^{\Delta}(t)+\int_{t_{0}}^{t} u^{\Delta}(s) \Delta s=0 \Longleftrightarrow u^{\Delta}(t)+u(t)=u\left(t_{0}\right) .
$$

We may notice that equation (31) cannot be written in form (13); hence, it is not self-adjoint. Indeed, notice that (31) is a first order dynamic equation while from Remark 18 one obtains a second order dynamic equation. Following Theorem 16 (see Remark 17), we conclude that (28) is not an Euler-Lagrange equation.

Now we consider the particular case of Theorem 16 when $\mathbb{T}=\mathbb{R}$ and $y \in C^{2}\left(\left[t_{0}, t_{1}\right] ; \mathbb{R}\right)$. In this case our operator $[\cdot]_{\mathbb{T}}$ of (14) has the form $[y]_{\mathbb{R}}(t)=\left(t, y(t), y^{\prime}(t)\right)$, while condition (13) can be written as

$$
p(t) u^{\prime}(t)+\int_{t_{0}}^{t} q(s) u(s) d s=\text { const. }
$$

Corollary 20. If a given integrodifferential equation

$$
H\left(t, y(t), y^{\prime}(t)\right)+\int_{t_{0}}^{t} G\left(s, y(s), y^{\prime}(s)\right) d s=\text { const }
$$

is to be the Euler-Lagrange equation of a variational problem

$$
\mathscr{I}(y)=\int_{t_{0}}^{t_{1}} L\left(t, y(t), y^{\prime}(t)\right) d t
$$

(cf., e.g., [17]), then its equation of variation

$$
\begin{aligned}
\partial_{2} H[u]_{\mathbb{R}} & (t) u(t)+\partial_{3} H[u]_{\mathbb{R}}(t) u^{\prime}(t) \\
& +\int_{t_{0}}^{t} \partial_{2} G[u]_{\mathbb{R}}(s) u(s)+\partial_{3} G[u]_{\mathbb{R}}(s) u^{\prime}(s) d s=0
\end{aligned}
$$

must be self-adjoint, in the sense of Definition 12 with (13) given by (32).

Proof. The proof follows from Theorem 16 with $\mathbb{T}=\mathbb{R}$.

Now we consider the particular case of Theorem 16 when $\mathbb{T}=h \mathbb{Z}, h>0$. In this case our operator $[\cdot]_{\mathbb{V}}$ of (14) has the form

$$
[y]_{h \mathbb{Z}}(t)=\left(t, y(t+h), \Delta_{h} y(t)\right)=:[y]_{h}(t),
$$


where

$$
\Delta_{h} y(t)=\frac{y(t+h)-y(t)}{h} .
$$

For $\mathbb{T}=h \mathbb{Z}, h>0$, condition (13) can be written as

$$
p(t) \Delta_{h} u(t)+\sum_{k=\left(t_{0} / h\right)}^{(t / h)-1} h q(k h) u(k h+h)=\text { const. }
$$

Corollary 21. If a given difference equation

$$
\begin{aligned}
& H\left(t, y(t+h), \Delta_{h} y(t)\right) \\
& \quad+\sum_{k=\left(t_{0} / h\right)}^{(t / h)-1} h G\left(k h, y(k h+h), \Delta_{h} y(k h)\right)=\mathrm{const}
\end{aligned}
$$

is to be the Euler-Lagrange equation of a discrete variational problem

$$
\mathscr{I}(y)=\sum_{k=\left(t_{0} / h\right)}^{\left(t_{1} / h\right)-1} h L\left(k h, y(k h+h), \Delta_{h} y(k h)\right)
$$

(cf., e.g., [18]), then its equation of variation

$$
\begin{gathered}
\partial_{2} H[u]_{h}(t) u(t+h)+\partial_{3} H[u]_{h}(t) \Delta_{h} u(t) \\
+h \sum_{k=\left(t_{0} / h\right)}^{(t / h)-1}\left(\partial_{2} G[u]_{h}(k h) u(k h+h)\right. \\
\left.+\partial_{3} G[u]_{h}(k h) \Delta_{h} u(k h)\right)=0
\end{gathered}
$$

is self-adjoint, in the sense of Definition 12 with (13) given by (38).

Proof. The proof follows from Theorem 16 with $\mathbb{T}=h \mathbb{Z}$.

Finally, let us consider the particular case of Theorem 16 when $\mathbb{T}=\overline{q^{\mathbb{Z}}}=q^{\mathbb{Z}} \cup\{0\}$, where $q^{\mathbb{Z}}=\left\{q^{k}: k \in \mathbb{Z}, q>\right.$ $1\}$. In this case operator $[\cdot]_{\mathbb{T}}$ of $(14)$ has the form $[y]_{\bar{q}}^{\mathbb{Z}}(t)=$ $\left(t, y(q t), \Delta_{q} y(t)\right)=:[y]_{q}(t)$, where

$$
\Delta_{q} y(t)=\frac{y(q t)-y(t)}{(q-1) t} .
$$

For $\mathbb{\mathbb { T }}=\overline{q^{\mathbb{Z}}}, q>1$, condition (13) can be written as

$$
p(t) \Delta_{q} u(t)+(q-1) \sum_{s \in\left[t_{0}, t\right) \cap \mathbb{T}} s r(s) u(q s)=\text { const }
$$

(cf., e.g., [19]), where we use notation $r(t)$ instead of $q(t)$ in order to avoid confusion between the $q=$ const that defines the time scale and function $q(t)$ of (13).

Corollary 22. If a given q-equation

$$
\begin{aligned}
& H\left(t, y(q t), \Delta_{q} y(t)\right) \\
& +(q-1) \sum_{s \in\left[t_{0}, t\right) \cap \mathbb{T}} s G\left(s, y(q s), \Delta_{q} y(s)\right)=\text { const }
\end{aligned}
$$

$q>1$, is to be the Euler-Lagrange equation of a variational problem

$$
\mathscr{I}(y)=(q-1) \sum_{t \in\left[t_{0}, t_{1}\right) \cap \mathbb{T}} t L\left(t, y(q t), \Delta_{q} y(t)\right)
$$

$t_{0}, t_{1} \in \overline{q^{\mathbb{Z}}}$, then its equation of variation

$$
\begin{array}{r}
\partial_{2} H[u]_{q}(t) u(q t)+\partial_{3} H[u]_{q}(t) \Delta_{q} u(t) \\
+(q-1) \sum_{s \in\left[t_{0}, t\right) \cap \mathbb{T}} s\left(\partial_{2} G[u]_{q}(s) u(q s)\right. \\
\left.\quad+\partial_{3} G[u]_{q}(s) \Delta_{q} u(s)\right)=0
\end{array}
$$

is self-adjoint, in the sense of Definition 12 with (13) given by (43).

Proof. Choose $\mathbb{\mathbb { T }}=\overline{q^{\mathbb{Z}}}$ in Theorem 16.

The reader interested in the study of Euler-Lagrange equations for problems of the $q$-variational calculus is referred to $[16,20,21]$ and references therein.

\section{Discussion}

On an arbitrary time scale $\mathbb{T}$, it is easy to show equivalence between the integrodifferential equation (15) and the second order differential equation (47) below (Proposition 23). However, when we consider equations of variations of them, we notice that it is impossible to prove an equivalence between them on an arbitrary time scale. This impossibility is true even in the discrete time scale $\mathbb{Z}$. The main reason is the lack of chain rule on time scales ([2], Example 1.85). However, in $\mathbb{} \mathbb{R}=\mathbb{R}$ we can present this equivalence (Proposition 24).

Proposition 23. The integrodifferential equation (15) is equivalent to a second order delta differential equation

$$
W\left(t, y^{\sigma}(t), y^{\Delta}(t), y^{\Delta \Delta}(t)\right)=0 .
$$

Proof. Let (47) be a given second order differential equation. We may write it as a sum of two components

$$
W\langle y\rangle_{\mathbb{T}}(t)=F\langle y\rangle_{\mathbb{T}}(t)+G[y]_{\mathbb{T}}(t)=0 .
$$

Let $F\langle y\rangle_{\mathbb{T}}=H^{\Delta}[y]_{\mathbb{T}}$. Then,

$$
H^{\Delta}\left(t, y^{\sigma}(t), y^{\Delta}(t)\right)+G\left(t, y^{\sigma}(t), y^{\Delta}(t)\right)=0 .
$$

Integrating both sides of (49) from $t_{0}$ to $t$, we obtain the integrodifferential equation (15).

Let $\mathbb{T}$ be a time scale such that $\mu$ is delta differentiable. The equation of variation of a second order differential equation (47) is given by

$$
\begin{gathered}
\partial_{4} W\langle u\rangle_{\mathbb{T}}(t) u^{\Delta \Delta}(t)+\partial_{3} W\langle u\rangle_{\mathbb{T}}(t) u^{\Delta}(t) \\
+\partial_{2} W\langle u\rangle_{\mathbb{T}}(t) u^{\sigma}(t)=0 .
\end{gathered}
$$


Equation (50) is obtained by using the method presented in Remark 14.

On an arbitrary time scale it is impossible to prove the equivalence between the equation of variation (16) and (50). Indeed, after differentiating both sides of (16) and using the product rule given by Theorem 4 , we have

$$
\begin{aligned}
\partial_{2} H[u]_{\mathbb{T}} & (t) u^{\sigma \Delta}(t)+\partial_{2} H^{\Delta}[u]_{\mathbb{T}}(t) u^{\sigma \sigma}(t) \\
& +\partial_{3} H[u]_{\mathbb{T}}(t) u^{\Delta \Delta}(t)+\partial_{3} H^{\Delta}[u]_{\mathbb{T}}(t) u^{\Delta \sigma}(t) \\
& +\partial_{2} G[u]_{\mathbb{T}}(t) u^{\sigma}(t)+\partial_{3} G[u]_{\mathbb{T}}(t) u^{\Delta}(t)=0 .
\end{aligned}
$$

The direct calculations

(i) $\partial_{2} H[u]_{\mathbb{T}}(t) u^{\sigma \Delta}(t)=\partial_{2} H[u]_{\mathbb{T}}(t)\left(u^{\Delta}(t)+\mu^{\Delta}(t) u^{\Delta}(t)+\right.$ $\left.\mu^{\sigma}(t) u^{\Delta \Delta}(t)\right)$

(ii) $\partial_{2} H^{\Delta}[u]_{\mathbb{T}}(t) u^{\sigma \sigma}(t)=\partial_{2} H^{\Delta}[u]_{\mathbb{T}}(t)\left(u^{\sigma}(t)+\right.$ $\left.\mu^{\sigma}(t) u^{\Delta}(t)+\mu(t) \mu^{\sigma}(t) u^{\Delta \Delta}(t)\right)$

(iii) $\partial_{3} H^{\Delta}[u]_{\mathbb{T}}(t) u^{\Delta \sigma}(t)=\partial_{3} H^{\Delta}[u]_{\mathbb{T}}(t)\left(u^{\Delta}(t)+\mu(t) u^{\Delta \Delta}(t)\right)$

allow us to write (51) in form

$$
\begin{gathered}
{\left[\mu^{\sigma}(t) \partial_{2} H[u]_{\mathbb{T}}(t)+\mu(t) \mu^{\sigma}(t) \partial_{2} H^{\Delta}[u]_{\mathbb{T}}(t)\right.} \\
\left.+\partial_{3} H[u]_{\mathbb{T}}(t)+\mu(t) \partial_{3} H^{\Delta}[u]_{\mathbb{T}}(t)\right] u^{\Delta \Delta}(t) \\
+\left[\partial_{2} H[u]_{\mathbb{T}}(t)+\left(\mu(t) \partial_{2} H[u]_{\mathbb{T}}(t)\right)^{\Delta}\right. \\
\left.+\partial_{3} H^{\Delta}[u]_{\mathbb{T}}(t)+\partial_{3} G[u]_{\mathbb{T}}(t)\right] u^{\Delta}(t) \\
+\left[\partial_{2} H^{\Delta}[u]_{\mathbb{T}}(t)+\partial_{2} G[u]_{\mathbb{T}}\right] u^{\sigma}(t)=0 ;
\end{gathered}
$$

that is, using Theorem 5,

$$
\begin{aligned}
& u^{\Delta \Delta}(t)\left[\mu(t) \partial_{2} H[u]_{\mathbb{T}}(t)+\partial_{3} H[u]_{\mathbb{T}}(t)\right]^{\sigma} \\
&+u^{\Delta}(t) {\left[\partial_{2} H[u]_{\mathbb{T}}(t)+\left(\mu(t) \partial_{2} H[u]_{\mathbb{T}}(t)\right)^{\Delta}\right.} \\
&\left.+\partial_{3} H^{\Delta}[u]_{\mathbb{T}}(t)+\partial_{3} G[u]_{\mathbb{T}}(t)\right] \\
&+u^{\sigma}(t)\left[\partial_{2} H^{\Delta}[u]_{\mathbb{T}}(t)+\partial_{2} G[u]_{\mathbb{T}}(t)\right]=0 .
\end{aligned}
$$

We are not able to prove that the coefficients of (53) are the same as in (50), respectively. This is due to the fact that we cannot find the partial derivatives of (47), that is, $\partial_{4} W\langle u\rangle_{\mathbb{T}}(t)$, $\partial_{3} W\langle u\rangle_{\mathbb{T}}(t)$, and $\partial_{2} W\langle u\rangle_{\mathbb{T}}(t)$, from (49) because of lack of chain rule on an arbitrary time scale. The equivalence, however, is true for $\mathbb{T}=\mathbb{R}$.

Proposition 24. The equation of variation

$$
\begin{aligned}
\partial_{2} H[u]_{\mathbb{R}} & (t) u(t)+\partial_{3} H[u]_{\mathbb{R}}(t) u^{\prime}(t) \\
& +\int_{t_{0}}^{t} \partial_{2} G[u]_{\mathbb{R}}(s) u(s)+\partial_{3} G[u]_{\mathbb{R}}(s) u^{\prime}(s) d s=0
\end{aligned}
$$

is equivalent to the second order differential equation

$$
\begin{gathered}
\partial_{4} W\langle u\rangle_{\mathbb{R}}(t) u^{\prime \prime}(t)+\partial_{3} W\langle u\rangle_{\mathbb{R}}(t) u^{\prime}(t) \\
+\partial_{2} W\langle u\rangle_{\mathbb{R}}(t) u(t)=0 .
\end{gathered}
$$

Proof. We show that coefficients of (54) and (55) are the same, respectively. Let $\mathbb{T}=\mathbb{R}$. From (48) and relation $F\langle u\rangle_{\mathbb{R}}=$ $(d / d t) H[u]_{\mathbb{R}}$ we have

$$
\begin{aligned}
W\left(t, u(t), u^{\prime}(t), u^{\prime \prime}(t)\right)= & \frac{d}{d t} H\left(t, u(t), u^{\prime}(t)\right) \\
& +G\left(t, u(t), u^{\prime}(t)\right) .
\end{aligned}
$$

Using notation (14) and chain rule (which is only valid for $\mathbb{T}=\mathbb{R}$ ) we can calculate the following partial derivatives:

(i) $\partial_{2} W\langle u\rangle_{\mathbb{R}}(t)=(d / d t) \partial_{2} H[u]_{\mathbb{R}}(t)+\partial_{2} G[u]_{\mathbb{R}}(t)$,

(ii) $\partial_{3} W\langle u\rangle_{\mathbb{R}}(t)=\partial_{2} H[u]_{\mathbb{R}}(t)+(d / d t) \partial_{3} H[u]_{\mathbb{R}}(t)+$ $\partial_{3} G[u]_{\mathbb{R}}(t)$

(iii) $\partial_{4} W\langle u\rangle_{\mathbb{R}}(t)=\partial_{3} H[u]_{\mathbb{R}}(t)$.

After differentiation of both sides of (54) we obtain

$$
\begin{aligned}
\partial_{3} H[u]_{\mathbb{R}}(t) u^{\prime \prime}(t) & \\
+ & \left(\partial_{2} H[u]_{\mathbb{R}}(t)+\frac{d}{d t} \partial_{3} H[u]_{\mathbb{R}}(t)+\partial_{3} G[u]_{\mathbb{R}}(t)\right) u^{\prime}(t) \\
& +\left(\frac{d}{d t} \partial_{2} H[u]_{\mathbb{R}}(t)+\partial_{2} G[u]_{\mathbb{R}}(t)\right) u(t)=0
\end{aligned}
$$

Hence, the intended equivalence is proved.

Proposition 24 allows us to obtain the classical result of [12, Theorem II] as a corollary of our Theorem 16 . The absence of a chain rule on time scales (even for $\mathbb{T}=\mathbb{Z}$ ) implies that the classical approach of [12] fails on time scales. This is the reason why here we introduced a completely different approach to the subject based on the integrodifferential form. The case $\mathbb{T}=\mathbb{Z}$ was recently investigated in [11]. However, similar to [12], the approach of [11] is based on the differential form and cannot be extended to general time scales.

\section{Disclosure}

This work is part of the first author's Ph.D., which is carried out at the University of Aveiro under the Doctoral Programme Mathematics and Applications of Universities of Aveiro and Minho.

\section{Conflict of Interests}

The authors declare that there is no conflict of interests regarding the publication of this paper.

\section{Acknowledgments}

This work was partially supported by Portuguese funds through the Center for Research and Development in Mathematics and Applications (CIDMA) and The Portuguese Foundation for Science and Technology (FCT), within Project PEst-OE/MAT/UI4106/2014. Dryl was also supported by FCT through the Ph.D. Fellowship 
SFRH/BD/51163/2010 and Torres by FCT within Project OCHERA, PTDC/EEI-AUT/1450/2012, cofinanced by FEDER under POFC-QREN with COMPETE Reference FCOMP-01-0124-FEDER-028894. The authors are very grateful to two anonymous referees for valuable remarks and comments, which significantly contributed to the quality of the paper.

\section{References}

[1] S. Hilger, "Analysis on measure chains-a unified approach to continuous and discrete calculus," Results in Mathematics, vol. 18, no. 1-2, pp. 18-56, 1990.

[2] M. Bohner and A. Peterson, Dynamic Equations on Time Scales, Birkhäuser Boston, Boston, Mass, USA, 2001.

[3] R. Agarwal, M. Bohner, D. O'Regan, and A. Peterson, "Dynamic equations on time scales: a survey," Journal of Computational and Applied Mathematics, vol. 141, no. 1-2, pp. 1-26, 2002.

[4] M. Bohner and A. Peterson, Advances in Dynamic Equations on Time Scales, Birkhäuser Boston, Boston, Mass, USA, 2003.

[5] M. Dryl, A. B. Malinowska, and D. F. M. Torres, "A time-scale variational approach to inflation, unemployment and social loss," Control and Cybernetics Journal, vol. 42, no. 2, pp. 399418, 2013.

[6] L. Bourdin and E. Trélat, "Pontryagin maximum principle for finite dimensional nonlinear optimal control problems on time scales," SIAM Journal on Control and Optimization, vol. 51, no. 5, pp. 3781-3813, 2013.

[7] M. Dryl and D. F. M. Torres, "Necessary optimality conditions for infinite horizon variational problems on time scales," Numerical Algebra, Control and Optimization, vol. 3, no. 1, pp. 145-160, 2013.

[8] R. Almeida and D. F. M. Torres, "Isoperimetric problems on time scales with nabla derivatives," Journal of Vibration and Control, vol. 15, no. 6, pp. 951-958, 2009.

[9] M. Dryl and D. F. M. Torres, "The delta-nabla calculus of variations for composition functionals on time scales," International Journal of Difference Equations, vol. 8, no. 1, pp. 27-47, 2013.

[10] N. Martins and D. F. M. Torres, "Calculus of variations on time scales with nabla derivatives," Nonlinear Analysis. Theory, Methods \& Applications. An International Multidisciplinary Journal. Series A: Theory and Methods, vol. 71, no. 12, pp. e763e773, 2009.

[11] L. Bourdin and J. Cresson, "Helmholtz's inverse problem of the discrete calculus of variations," Journal of Difference Equations and Applications, vol. 19, no. 9, pp. 1417-1436, 2013.

[12] D. R. Davis, "The inverse problem of the calculus of variations in higher space," Transactions of the American Mathematical Society, vol. 30, no. 4, pp. 710-736, 1928.

[13] J. Cresson, A. B. Malinowska, and D. F. M. Torres, “Time scale differential, integral, and variational embeddings of Lagrangian systems," Computers \& Mathematics with Applications, vol. 64, no. 7, pp. 2294-2301, 2012.

[14] D. F. M. Torres, “The variational calculus on time scales," International Journal for Simulation and Multidisciplinary Design Optimization, vol. 4, no. 1, pp. 11-25, 2010.

[15] R. A. C. Ferreira, A. B. Malinowska, and D. F. M. Torres, "Optimality conditions for the calculus of variations with higher-order delta derivatives," Applied Mathematics Letters, vol. 24, no. 1, pp. 87-92, 2011.
[16] R. A. C. Ferreira and D. F. M. Torres, "Necessary optimality conditions for the calculus of variations on time scales," 2007, http://arxiv.org/abs/0704.0656 .

[17] B. van Brunt, The Calculus of Variations, Universitext, Springer, New York, NY, USA, 2004.

[18] N. R. O. Bastos, R. A. C. Ferreira, and D. F. M. Torres, "Discretetime fractional variational problems," Signal Processing, vol. 91, no. 3, pp. 513-524, 2011.

[19] M. R. Segi Rahmat, "On some $(q, h)$-analogues of integral inequalities on discrete time scales," Computers \& Mathematics with Applications, vol. 62, no. 4, pp. 1790-1797, 2011.

[20] A. B. Malinowska and D. F. M. Torres, Quantum Variational Calculus, SpringerBriefs in Electrical and Computer Engineering: Control, Automation and Robotics, Springer, New York, NY, USA, 2014.

[21] N. Martins and D. F. M. Torres, "Higher-order infinite horizon variational problems in discrete quantum calculus," Computers \& Mathematics with Applications, vol. 64, no. 7, pp. 2166-2175, 2012. 


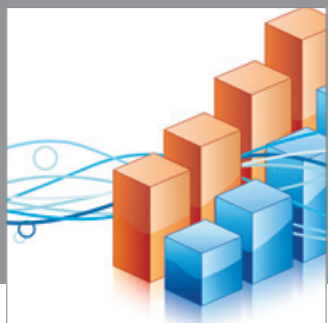

Advances in

Operations Research

mansans

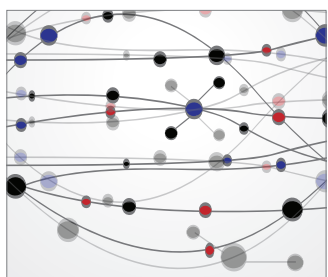

The Scientific World Journal
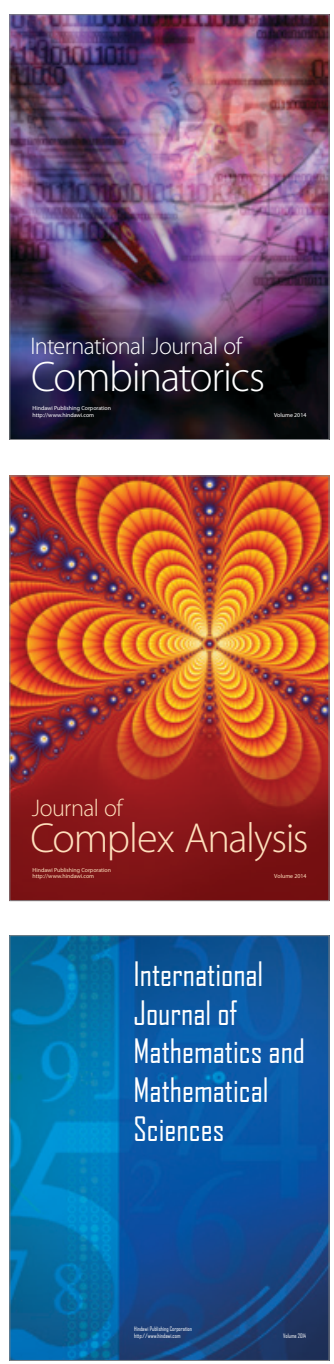
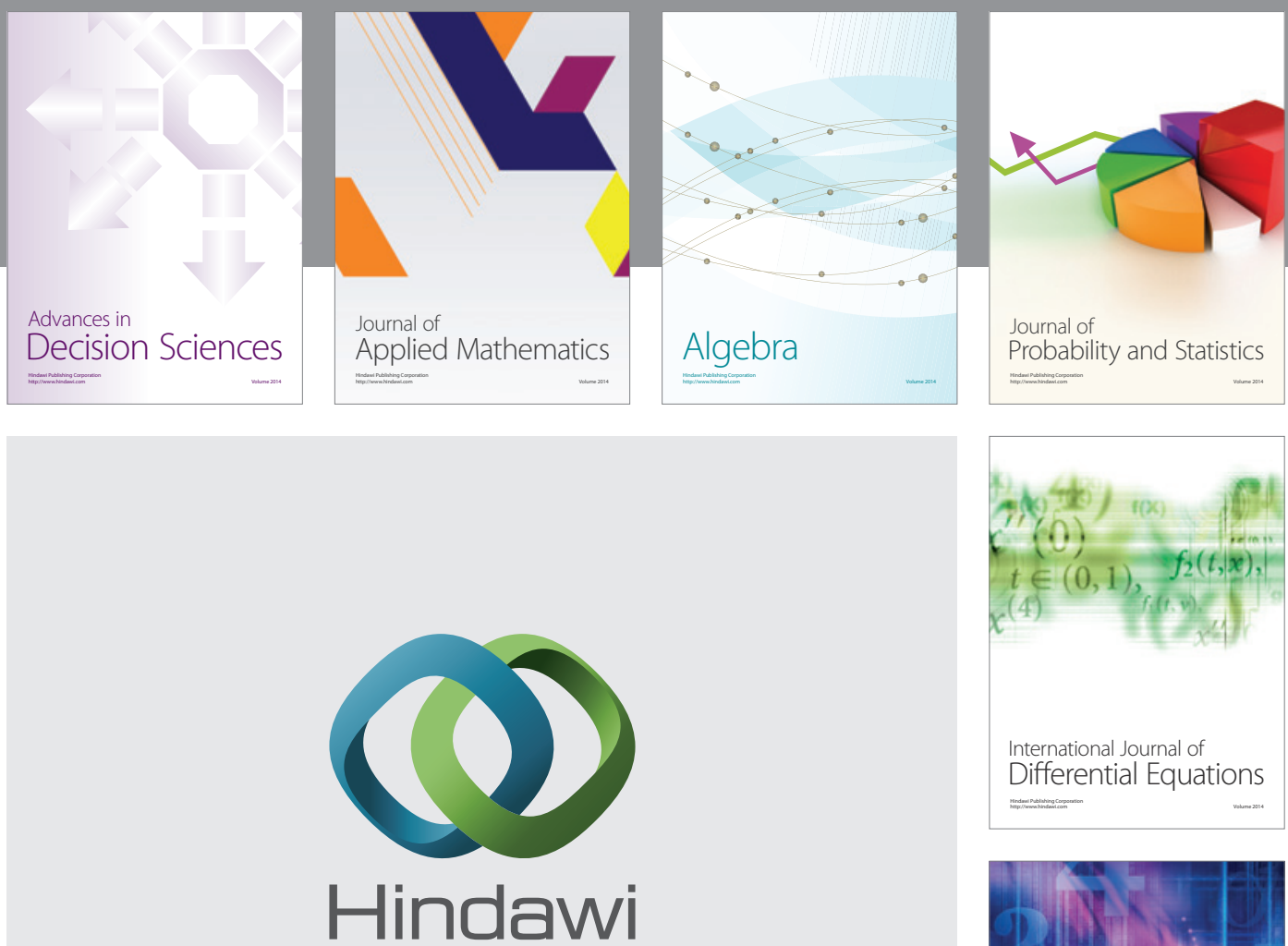

Submit your manuscripts at http://www.hindawi.com
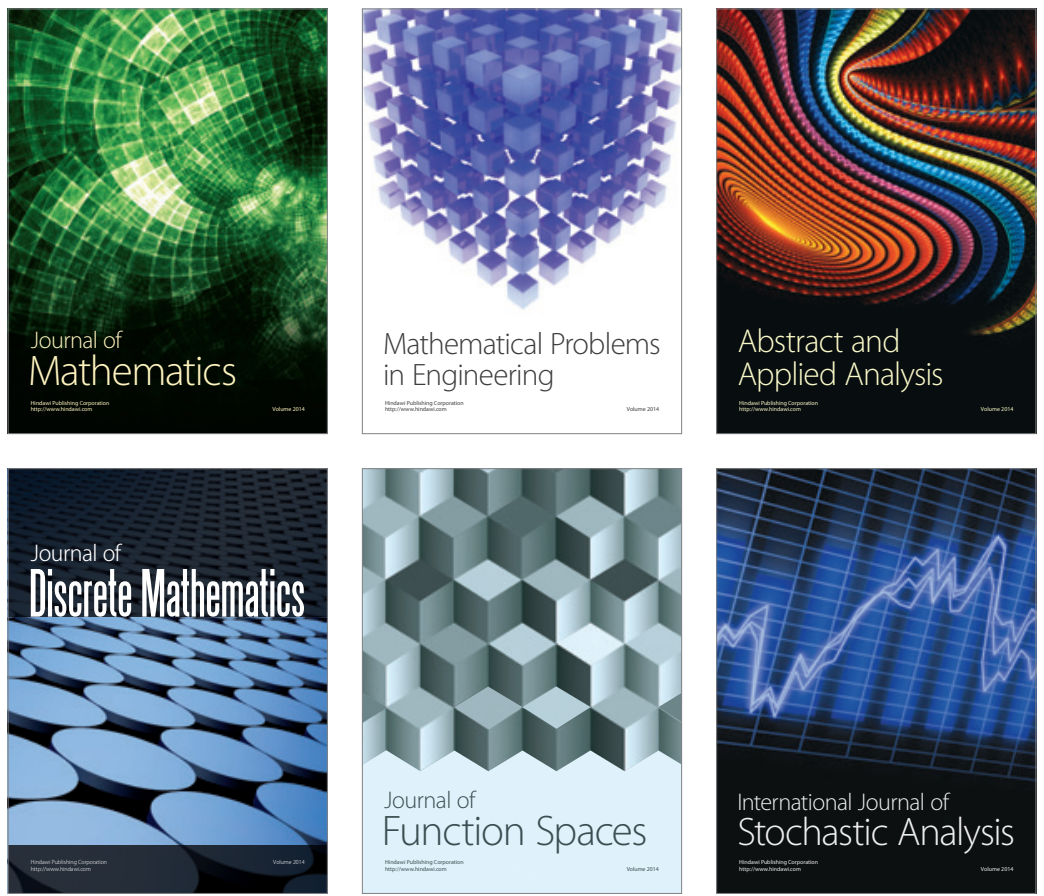

Journal of

Function Spaces

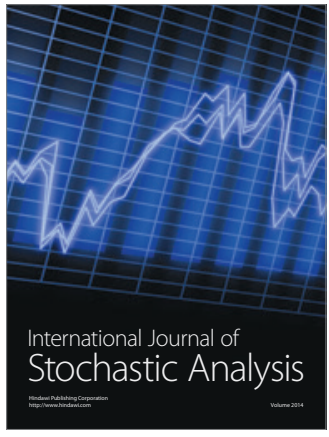

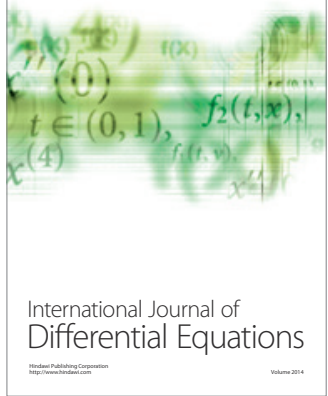
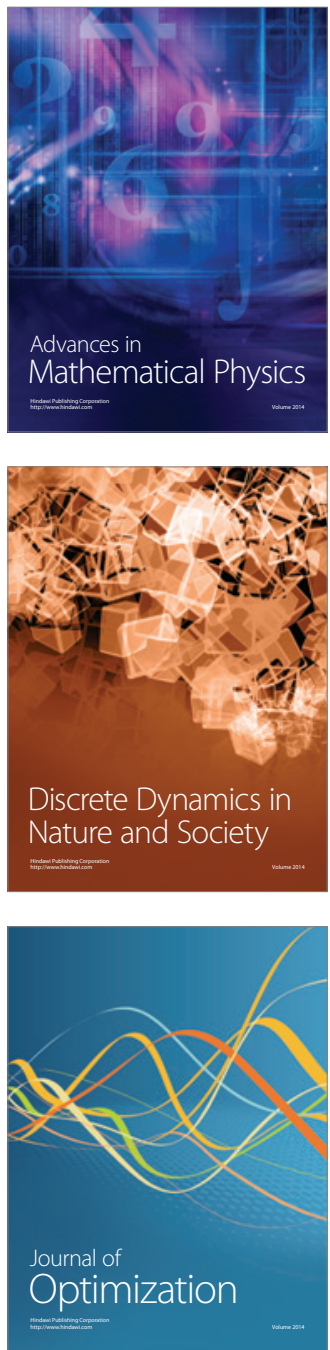